\title{
Radiation forces on a Rayleigh particle by highly focused radially polarized beams modulated by DVL
}

\author{
Ruili Zhang , Ziyang Chen, and Jixiong Pu* \\ College of Information Science and Engineering, Fujian Provincial Key Laboratory of \\ Light Propagation and Transformation, Huaqiao University, Xiamen 361021, China \\ P. H. Jones \\ Department of Physics and Astronomy, University College London, Gower Street, \\ London, \\ WC1E 6BT, United Kingdom
}

\begin{abstract}
The intensity and the radiation forces acting on a Rayleigh particle near the focus of completely coherent radially polarized beams whose phase are modulated by a Devil's vortex-lens are studied. The influence of the structure of Devil's vortex-lens on the radiation force distribution is analyzed. It is found by numerical simulations that the modulated beams show a clear advantage over the unmodulated highly focused radially polarized beams, as the modulated beam can simultaneously trap and manipulate the multiple Rayleigh particles, while the unmodulated beam can trap only one particle under the same condition.
\end{abstract}

OCIS codes: (030.1640) Coherence; (260.5430) Polarization; (260.6042) Singular optics; (350.4855) Optical tweezers or optical manipulation.

\section{INTRODUCTION}

Since the original paper on dislocations in wave trains by Nye and Berry ${ }^{[1]}$, optical vortices have attracted increasing attention due to their broad applications in creating micromechanical pumps ${ }^{[2]}$, storing quantum information ${ }^{[3]}$, enhancing microscopy ${ }^{[4]}$. As is well known, optical vortices extended the capabilities of conventional optical traps ${ }^{[5,6]}$ due to the orbital angular momentum of light ${ }^{[7,8]}$. One common optical device to produce optical vortices is the spiral phase plates (SPP). Spiral phase plates can be combined with fractal zone plates ${ }^{[9]}$ to produce a sequence of focused optical vortices along the propagation direction ${ }^{[10]}$. Recently, a phase-only Devil's vortex-lens (DVL) modulated by a helical phase structure ${ }^{[11]}$ was proposed, which is able to generate a sequence of focused vortices surrounding the major foci inside a single major fractal focus. 
The radially polarized beam can generate a strong longitudinal electric field at the focal point ${ }^{[12]}$ when focused by a high-numerical-aperture (NA) focusing system, which can be applied in improving resolution of microscopy ${ }^{[13]}$, enhancing laser cutting ability ${ }^{[14]}$. Analytical calculations by Chen et al. ${ }^{[15]}$ showed that the radially polarized beams are superior to the linearly polarized beams in the Rayleigh regime in terms of their ability to trap. Zhan calculated the radiation forces on metallic Rayleigh particles using a highly focused ideal radially polarized beam ${ }^{[16]}$. Ahluwalia et al. investigated experimentally the polarization-induced torque of radially polarized beam acting on anisotropic microparticles ${ }^{[17]}$.

In this paper, we study the properties of highly focused completely coherent radially polarized beams modulated by DVL. Based on the vector Debye integral ${ }^{[18]}$, the theoretical expressions of focused field are derived. The radiation forces acting on Rayleigh particles are calculated by using the Rayleigh scattering theory. A bright spot can be generated by matching the topological charge and function order of DVL, which can be applied in three-dimensionally trapping a Rayleigh dielectric sphere with a refractive index larger than that of the ambient.

\section{THEORETICAL ANALYSIS}

A devil's lens (DL) is a rotationally symmetric diffractive lens whose phase profile is designed from a staircase function based on the Cantor function ${ }^{[12]}$ mathematically. It is defined in the domain $[0,1]$ as

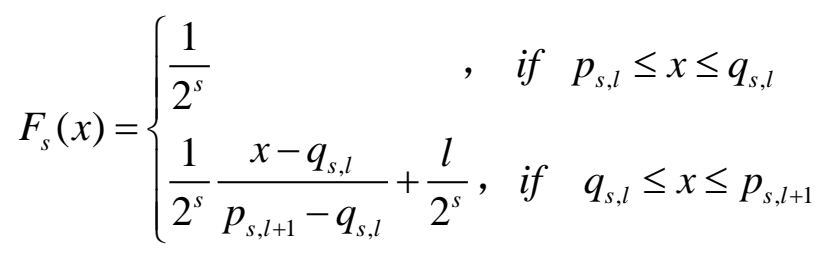

Being $F_{S}(0)=0$ and $F_{S}(1)=1$, at the disjoint gaps intervals $\left[p_{s, l}, q_{s, l}\right]$ defined by a particular Cantor function $F_{S}(x)$ the phase shift is $-l 2 \pi$, with $l=1, \ldots, 2^{\mathrm{s}}-1$, a DL is a pure-phase diffractive optical element whose transmittance is defined by ${ }^{[19]}$

$$
Q(\varsigma)=\exp \left[-i 2^{s+1} \pi F_{s}(\varsigma)\right]
$$

where

$$
\varsigma=\left(\frac{\sin \theta}{\sin \alpha}\right)^{2}
$$


where $\mathrm{R}$ is the lens radius, and a DVL can be simply constructed by adding the phase variation $m \phi$, where $m$ is the topological charge and $\phi$ is the azimuthal angle. The transmittance of a DVL can be expressed as ${ }^{[20]}$

$$
T(\theta, \phi)=Q(\theta) \cdot \exp [i m \phi]
$$

In this study, we investigate the intensity and the force in the vicinity of focus of a highly NA focusing system combined with DVL under the irradiance of radially polarized beams. Figure 1 illustrates the geometry of the tight focusing system. The separation between the geometrical focal plane of the lens and the interface of two media is $d$, and $f$ being the focal length of the lens. The refractive index of the two media are $n_{1}$ and $n_{2}$, the incident and refractive angles are $\theta_{1}, \theta_{2}$, respectively.

It is assumed that the field in the source plane is a completely coherent radially polarized Bessel-Gauss beam, which can be written as ${ }^{[21]}$ :

$$
A(\theta)=E_{0} \exp \left[-\beta^{2}\left(\frac{\sin \theta}{\sin \alpha}\right)^{2}\right] J_{1}\left(2 \beta \frac{\sin \theta}{\sin \alpha}\right)
$$

where $w_{0}$ is the beam width, $E_{0}$ is a constant.

Supposed the input power is $P$, the intensity distribution in the source plane $I_{0}(r)$ can be calculated as

$$
P=\iint I_{0}(r) d s
$$

and

$$
E_{0}=\sqrt{\frac{3.54 P \mathrm{e}}{\pi f^{2} \sin ^{2} \alpha}}
$$

Where $\mathrm{e}$ is the natural logarithm, $\alpha$ is the maximal NA angle.

We have the pupil apodization function at objective aperture surface:

$$
A\left(\theta_{1}\right)=\sqrt{\frac{3.54 P e}{\pi f^{2} \sin ^{2} \alpha}} \exp \left(\frac{-\sin ^{2} \theta_{1}}{\sin ^{2} \alpha}\right) J_{1}\left(\frac{2 \sin \theta_{1}}{\sin \alpha}\right)
$$

According to the Richards-Wolf vector diffraction theory ${ }^{[18]}$ and the sine condition $r=f \cdot \sin \left(\theta_{1}\right)^{[22]}$, when a radially polarized beam modulated by DVL is highly focused, the electric field $E(\rho, \varphi, z)$ in the focal region can be obtained by the following expression: 


$$
\begin{aligned}
E(\rho, \varphi, z)= & {\left[\begin{array}{c}
E_{x} \\
E_{y} \\
E_{z}
\end{array}\right]=-i \frac{K}{\pi} \int_{0}^{\alpha} \int_{0}^{2 \pi} \sin \theta_{1} \sqrt{\cos \theta_{1}} A\left(\theta_{1}\right) T\left(\theta_{1}, \phi\right) \exp \left[-i k_{0} \Phi\left(\theta_{1}, \theta_{2}\right)\right] t_{p} } \\
& \times \exp \left(-i k_{1} z \cos \theta_{1}-\rho \sin \theta_{1} \cos (\phi-\varphi)\right)\left[\begin{array}{l}
\cos \theta_{1} \cos \phi \\
\cos \theta_{1} \sin \phi \\
\sin \theta_{1}
\end{array}\right] \mathrm{d} \phi \mathrm{d} \theta_{1}
\end{aligned}
$$

Where $\rho, \varphi$, and $z$ are the cylindrical coordinates of an observation point, $k_{i}=n_{i} k_{0}$ is the wave vector, the maximal NA angle is $\alpha=\sin ^{-1}\left(\mathrm{NA} / n_{1}\right), K$ is a constant $K=\frac{\pi f \sqrt{n_{1}}}{\lambda}$, other parameter $T\left(\theta_{1}, \phi\right)$ is the transmittance of a DVL. The amplitude transmission coefficient $t_{p}$ perpendicular to the polarization direction can be expressed as:

$$
t_{p}=\frac{2 \sin \theta_{2} \cos \theta_{1}}{\sin \left(\theta_{1}+\theta_{2}\right) \cos \left(\theta_{1}-\theta_{2}\right)}
$$

and $\Phi\left(\theta_{1}, \theta_{2}\right)$ is aberration function related to the refractive index $n_{1}$ and $n_{2}$ as follows:

$$
\Phi\left(\theta_{1}, \theta_{2}\right)=-d\left(n_{1} \cos \theta_{1}-n_{2} \cos \theta_{2}\right)
$$

The Cartesian electric field expressions ${ }^{[22,23]}$ near focus formed by highly focused radially polarized beam modulated by DVL passing an interface of two media are:

$$
E(\rho, \varphi, z)=\left[\begin{array}{c}
E_{x}(\rho, \varphi, z) \\
E_{y}(\rho, \varphi, z) \\
E_{z}(\rho, \varphi, z)
\end{array}\right]=-i^{m+1} \mathrm{~K} E_{0}\left[\begin{array}{c}
i\left(I_{m+1} e^{i \varphi}-I_{m-1} e^{-i \varphi}\right) \\
I_{m+1} e^{i \varphi}+I_{m-1} e^{-i \varphi} \\
2 I_{m}
\end{array}\right] e^{i m \varphi}
$$

where

$$
\begin{aligned}
I_{m}(\rho, z)= & \int_{0}^{\alpha} \exp \left[-i k_{0} \Phi\left(\theta_{1}, \theta_{2}\right)\right] A\left(\theta_{1}\right) q\left(\theta_{1}\right) \sqrt{\cos \theta_{1}} \sin \theta_{1} t_{p} \sin \theta_{2} \\
& \times J_{m}\left(k_{1} \rho \sin \theta_{1}\right) \exp \left(-i k_{2} z \cos \theta_{2}\right) \mathrm{d} \theta_{1} \\
I_{m \pm 1}(\rho, z)= & \int_{0}^{\alpha} \exp \left[-i k_{0} \Phi\left(\theta_{1}, \theta_{2}\right)\right] A\left(\theta_{1}\right) q\left(\theta_{1}\right) \sqrt{\cos \theta_{1}} \sin \theta_{1} t_{p} \cos \theta_{2} \\
& \times J_{m \pm 1}\left(k_{1} \rho \sin \theta_{1}\right) \exp \left(-i k_{2} z \cos \theta_{2}\right) \mathrm{d} \theta_{1}
\end{aligned}
$$

Based on the equation (12), (13), the radial and azimuthal components of the electric field in the focal plane can be obtained by using the following transformations:

$$
\begin{gathered}
E_{\rho}(\rho, \varphi, z)=E_{x}(\rho, \varphi, z) \cos \varphi+E_{y}(\rho, \varphi, z) \sin \varphi \\
E_{\varphi}(\rho, \varphi, z)=-E_{x}(\rho, \varphi, z) \sin \varphi+E_{y}(\rho, \varphi, z) \cos \varphi
\end{gathered}
$$


Then the intensity distribution in the focal region can be expressed as:

$$
I(\rho, \varphi, z)=\left|E_{\rho}(\rho, \varphi, z)\right|^{2}+\left|E_{\varphi}(\rho, \varphi, z)\right|^{2}+\left|E_{z}(\rho, \varphi, z)\right|^{2}
$$

We choose a Rayleigh particle as the target particle. Under this condition, a small dielectric sphere can be treated as a point dipole ${ }^{[24]}$. According to the Rayleigh scattering theory, the radiation force exerted on this dipole can be divided into two components ${ }^{[25]}$ : scattering force and gradient force. Assuming a homogeneous Rayleigh microsphere with refractive index $n_{3}$ and radius $a$, the scattering force can be expressed as:

$$
F_{s c a t}(\rho, \varphi, z)=\frac{n_{2}}{c} C_{p r} I(\rho, \varphi, z) \mathbf{e}_{\mathbf{z}}
$$

where $c=\frac{1}{\sqrt{\varepsilon_{0} \mu_{0}}}, \quad C_{p r}=\frac{8}{3} \pi(k a)^{4} a^{2}\left(\frac{\mathrm{H}^{2}-1}{\mathrm{H}^{2}+2}\right)^{2}, \mathrm{H}=n_{2} / n_{3}, \mathrm{H}$ is the relative refractive index. Highly focused radially polarized beams can produce strong longitudinal electric field due to the depolarization ${ }^{[26]}$. The gradient forces along the axial and radial direction are given by ${ }^{[27]}$

$$
\begin{gathered}
F_{\text {grad }}(\rho, \varphi, z)=\frac{2 \pi n_{2} a^{3}}{c}\left(\frac{\mathrm{H}^{2}-1}{\mathrm{H}^{2}+2}\right) \frac{\partial}{\partial z} I(\rho, \varphi, z) \\
F_{\text {grad }}(\rho, \varphi, z)=\frac{2 \pi n_{2} a^{3}}{c}\left(\frac{\mathrm{H}^{2}-1}{\mathrm{H}^{2}+2}\right) \frac{\partial}{\partial r} I(\rho, \varphi, z)
\end{gathered}
$$

\section{THE NUMERICAL SIMULATION}

In this part, the particle to be trapped is assumed to be immersed in water with refractive index $n_{2}$. In the numerical calculations, we choose the following parameters: $f=0.0025 \mathrm{~m}, w_{0}=0.0025 \mathrm{~m}, \quad R=0.0025 \mathrm{~m}$, $a=30 \mathrm{~nm}, n_{1}=1, n_{2}=1.332, n_{3}=1.592, N A=1, d=\lambda, \lambda=633 \mathrm{~nm}$, and the total incident power $P$ is $2 W$.

Figure 2 shows the electric field intensity with the change of function order $\mathrm{s}$. A single focal spot is produced with $\mathrm{s}=0$, and multiple focal spots are produced with larger s, as present in figure 2(a), (b), (c). In particular, the position of the major focal point is not located in $\mathrm{z}=0$ plane with $\mathrm{s}=1$.

Figure 3 shows the intensity in the vicinity of focus formed by highly focused radially polarized beam with different topological charge $m$. The intensity distributes along the axis is strongest with $m=1$. The on-axis intensity is zero when $\mathrm{m}$ is larger than 2 . It keeps the property of DVL as shown in Ref. ${ }^{[20]}$, each focus transforms into a vortex and a chain of doughnut shaped foci is generated. The tightly focused field can be used to trap multiple target particles, which is shown in the next section. 
In Figure 4, 5, 6, we study the transverse gradient force, the on-axis longitudinal gradient force and the scattering force acting on the Rayleigh particle by highly focused radially polarized beams modulated by DVL. From Figure 4(c), (d), the on-axis longitudinal gradient forces in the optimal capturing positions of $\mathrm{z}=1 \lambda$ are higher than the scattering force that destabilize the trap ${ }^{[26]}$, which satisfies the condition $\Gamma=F_{\text {grand }} / F_{\text {scal }} \geq 1$. It can be clearly found in Figure. 5 and Figure. 6 that a Rayleigh particle whose refractive index is larger than that of the ambient environment can be trapped and captured at the focal points of the major and the subsidiary near it. The best capturing positions are $z=3.5 \lambda, z=6 \lambda, z=11 \lambda$ in Figure 5 and $z=4 \lambda, z=8 \lambda, z=11 \lambda$ in Figure6.

The thermal fluctuation from the surrounding medium is called Brownian motion, which can have a strong affect in capturing the extremely small particles from the perspective of principle. Based on the fluctuation-dissipation theorem of Einstein, a formula ${ }^{[28]}$ to calculate the Brownian force is given by $\left|F_{B}\right|=\left(12 \pi \kappa a k_{B} T\right)^{1 / 2}$, where $\kappa$ represents the viscosity of the medium (in this paper, for water $\kappa=7.977 \times 10^{-4}, T=300 \mathrm{~K}$ ), and $k_{B}$ is the Boltzmann constant. The magnitude of the Brownian force is to be $1.933 \times 10^{-3} \mathrm{PN}$ under these conditions, which is smaller compared with the values of the scattering and the gradient components of the radiation forces in Figure 4, 5, 6, therefore, the Brownian motion can be obviously overcome.

The gradient force curves in Figure 4, 5, 6 show the number of the equilibrium positions of particles in the trap. We research the magnitude of Brownian motion, the result show that the influence of Brownian motion can be neglected. For DVL with the parameters of $s=1, m=0$, there are three positions $\mathrm{z}=3.5 \lambda, \mathrm{z}=6 \lambda, \mathrm{z}=11 \lambda$, where the gradient force is much larger than the scatting force, as presented in Figure 5. Similar results can be found in Figure 6, where the equilibrium positions lie in the plane of $z=4 \lambda, z=8 \lambda, z=11 \lambda$. Clearly, stable trap can be realized in these positions. Previous studies show that even though there is only one equilibrium position on the beam axis, the beam might trap multiple microparticles simultaneously ${ }^{[29-31]}$. Our research show that multiple particles may trap simultaneously in different locations by introducing a DVL into the focusing system.

\section{CONCLUSION}

In conclusion, the radiation forces on a dielectric spherical particle induced by highly focused radially polarized beams modulated by DVL have been numerically studied in the Rayleigh scattering regime. 
Simulation results suggested that the radially polarized vector beams modulated by DVL shows advantage over the conventional highly focused radial polarization beam in trapping microparticles, the former can simultaneously trap and manipulate multiple particles, but the latter can only trap one particle by using the highly focused radially polarized beam, even under the same condition. It is expected to pave the way for multiple focal plane trapping.

\section{ACKNOWLEDGMENTS}

This work was supported by the National Natural Science Foundation of China (NSFC) (11304104, and 61178015), and Promotion Program for Young and Middleaged Teacher in Science and Technology Research of Huaqiao University (ZQN-PY209).

* jixiong@hqu.edu.cn

\section{REFERENCES:}

1. J. F. Nye and M. V. Berry, "Dislocations in Wave Trains," Proc. R. Soc. London Ser. A . $336,165-190(1974)$.

2. K. Ladavac and D. G. Grier, "Microoptomechanical pumps assembled and driven by holographic optical vortex arrays," Opt. Express. 12, 1144-1149 (2004).

3. J. Leach, B. Jack, J. Romero, A. K. Jha, A. M. Yao, S. F. Arnold, D. G. Ireland, R. W. Boyd, S. M. Barnett, and M. J. Padgett, "Quantum Correlations in Optical Angle-Orbital Angular Momentum Variables," Science. 329, 662-665 (2010).

4. S. Ferhapter, A. Jesacher, S. Bernet, and M. R. Marte, "Spiral Phase Contrast Imaging in Microscopy," Opt. Express. 13, 689-694 (2005).

5. K. T. Gahagan and G. A. Swartzlander, "Optical vortex trapping of particles," Opt. Lett. 21, 827-829 (1996).

6. H. He, M. E. J. Friese, N. R. Heckenberg, and H. Rubinsztein-Dunlop, "Direct Observation of Transfer of Angular Momentum to Absorptive Particles from a Laser Beam with a Phase Singularity," Phys. Rev. Lett. 75, 826-829 (1995).

7. F. S. Roux, "Distribution of angular momentum and vortex morphology in optical beams," Opt. Commun. 242, 45-55 (2004).

8. G. Gbur, T. D. Visser, "Phase singularities and coherence vortices in linear optical systems," Opt. Commun. 259, 428-435 (2006).

9. S. G, F. WD, M. JA, "Fractal zone plates," Opt. Lett. 28 , 971-973 (2003).

10. S. H. Tao, X. C. Yuan, J. Lin, and R. E. Burge, "Sequence of focused optical vortices generated by a spiral fractal zone plate," Appl. Phys. Lett. 89, 031105 (2006). 
11. J. A. Monsoriu, W. D. Furlan, G. Saavedra, and F. Giménez, "Devil's lenses," Opt. Express .15 , 13858-13864 (2007).

12. K. Youngworth, and T. Brown, "Focusing of high numerical aperture cylindricalvector beams," Opt. Express. 7, 77-87 (2000).

13. S. Quabis, R. Dorn, M. Eberler, O. Glöckl and G. Leuchs, "Focusing light to a tighter spot1," Opt. Commun. 179, 1-7 (2000).

14. N.A.V, and N. V. G, "Laser beams with axially symmetric polarization," J. Phys. D: Appl. Phys.33, 1817-1822 (2000).

15. J. Chen, J. Ng, S. Liu, and Z. Lin, “Analytical calculation of axial optical force on a Rayleigh particle illuminated by Gaussian beams beyond the paraxial approximation," Phys. Rev. E. 80, 026607 (2009).

16. Q. Zhan, "Trapping metallic Rayleigh particles with radial polarization," Opt. Express. 12, 3377-3382 (2004).

17. B. P. S. Ahluwalia, X. C. Yuan, K. J. Moh, and J. Bu, Appl. Phys. Lett. 91, 171102 (2007).

18. B. Richards, E. Wolf, "Electromagnetic Diffraction in Optical Systems. II. Structure of the Image Field in an Aplanatic System," Proc R Soc A. 253, 358379 (1959).

19. C. Alejandro, S. R. Sergio, M. Leon, and Lluis, "Generation of programmable 3D optical vortex structures through devil's vortex-lens arrays," Appl. Optics. 52, 5822-5829 (2013).

20. F. W. D, G. Fernando, and C. Arnau, "Devil's vortex-lenses," Opt. Express. 17, 21891-21896 (2009).

21. K. S. Youngworth and T. G. Brown, "Focusing of high numerical aperture cylindrical-vector beams," Opt. Express. 7, 77-87 (2000).

22. M. Gu, Advanced Optical Imaging Theory (Springer-Verlag,1999).

23. S. C. Tidwell, G. H. Kim, and W. D. Kimura, "Efficient radially polarized laser beam generation with a double interferometer," Appl. Optics. 32, 5222-5229 (1993).

24. Y. Zhang, T. Suyama, and B. Ding, "Longer axial trap distance and larger radial trap stiffness using a double-ring radially polarized beam," Opt. Lett. 35, 12811283 (2010).

25. Y. Harada and T. Asakura, "Radiation forces on a dielectric sphere in the Rayleigh scattering regime," Opt. Commun. 124, 529-541 (1996).

26. Q. Zhan, "Trapping metallic Rayleigh particles with radial polarization: reply to comment," Opt. Express. 20, 6058-6059 (2012).

27. L. N. Ng, B. J. Luff, M. N. Zervas, and J. S. Wilkinson, "Forces on a Rayleigh Particle in the Cover Region of a Planar Waveguide," J. Lightwave Technol. 18, 388- 400 (2000).

28. K. Okamoto and S. Kawata, "Radiation force exerted on subwavelength particles near a nanoaperture," Phys. Rev. Lett. 83, 4534-4537 (1999). 
29. S. Xu, Y. Li, and L. Lou, "Axial optical trapping forces on two particles trapped simultaneously by optical tweezers," Appl. Opt. 44, 2667(2005).

30. L. Martin, and A. Jochen, "Trapping multiple particles in single optical tweezers," Opt. Commun. 281,135-140(2008).

31. F. Liu, Z. Zhang, Y. Wei, Q. Zhang, T. Cheng, and X. Wu. "Photophoretic trapping of multiple particles in tapered-ring optical field," Opt. Exp. 22, 23716 (2014). 


\section{List of figure captions:}

Fig. 1 schematics of highly focused radially polarized beams modulated by DVL.

Fig. 2 the intensity distribution produced by highly focused radially polarized beams modulated by DVL lens with the change of function order $\mathrm{s}$. (a)s $=0, \mathrm{~m}=1$; (b) $\mathrm{s}=1, \mathrm{~m}=1$; (c) $\mathrm{s}=2, \mathrm{~m}=1$, (d) the on-axis intensity distribution.

Fig.3 the intensity distribution produced by highly focused radially polarized beams modulated by DVL lens with the change of topological charge.(a) $\mathrm{m}=0, \mathrm{~s}=1$; (b) $\mathrm{m}=1, \mathrm{~s}=1$; (c) $\mathrm{m}=2, \mathrm{~s}=1$, (d) the on-axis intensity distribution.

Fig.4 The radiation force distribution by highly focused radially polarized beams modulated by DVL. (a) the radial gradient force in the plane of $z=1 \lambda$; (b) the axial gradient force ; (c) scattering force. the other parameters are $\mathrm{s}=0, \mathrm{~m}=1$.

Fig.5 The radiation force distribution by highly focused radially polarized beams modulated by DVL. (a) the radial gradient force in the plane of $z=3.5 \lambda, z=6 \lambda$, $\mathrm{z}=11 \lambda$; (b) the axial gradient force; (c) scattering force. the other parameters are $\mathrm{s}=1, \mathrm{~m}=0$.

Fig.6 The radiation force distribution by highly focused radially polarized beams modulated by DVL, (a) the radial gradient force in the plane of $z=4 \lambda, z=8 \lambda$, $\mathrm{z}=11 \lambda$; (b) the axial gradient force; (c) scattering force. the other parameters are $\mathrm{s}=1, \mathrm{~m}=1$. 


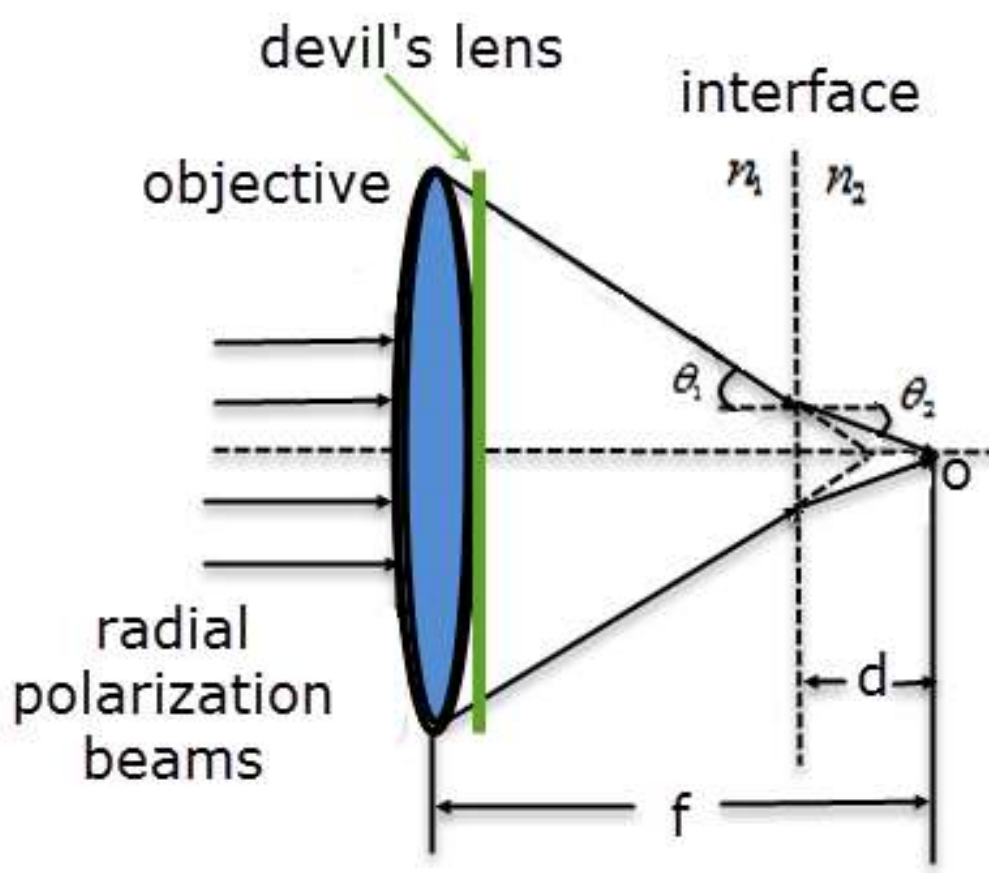

Fig.1 

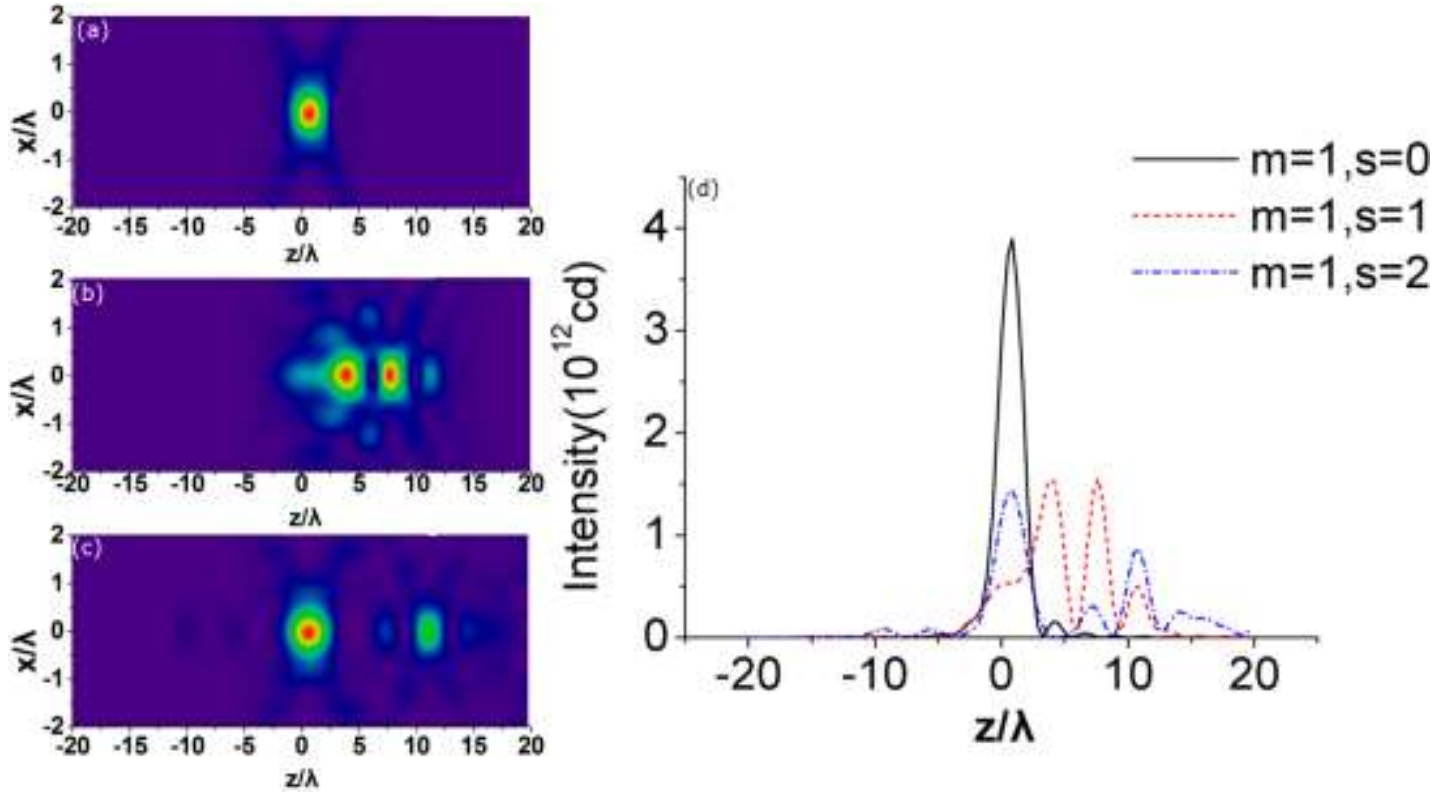

Fig.2 

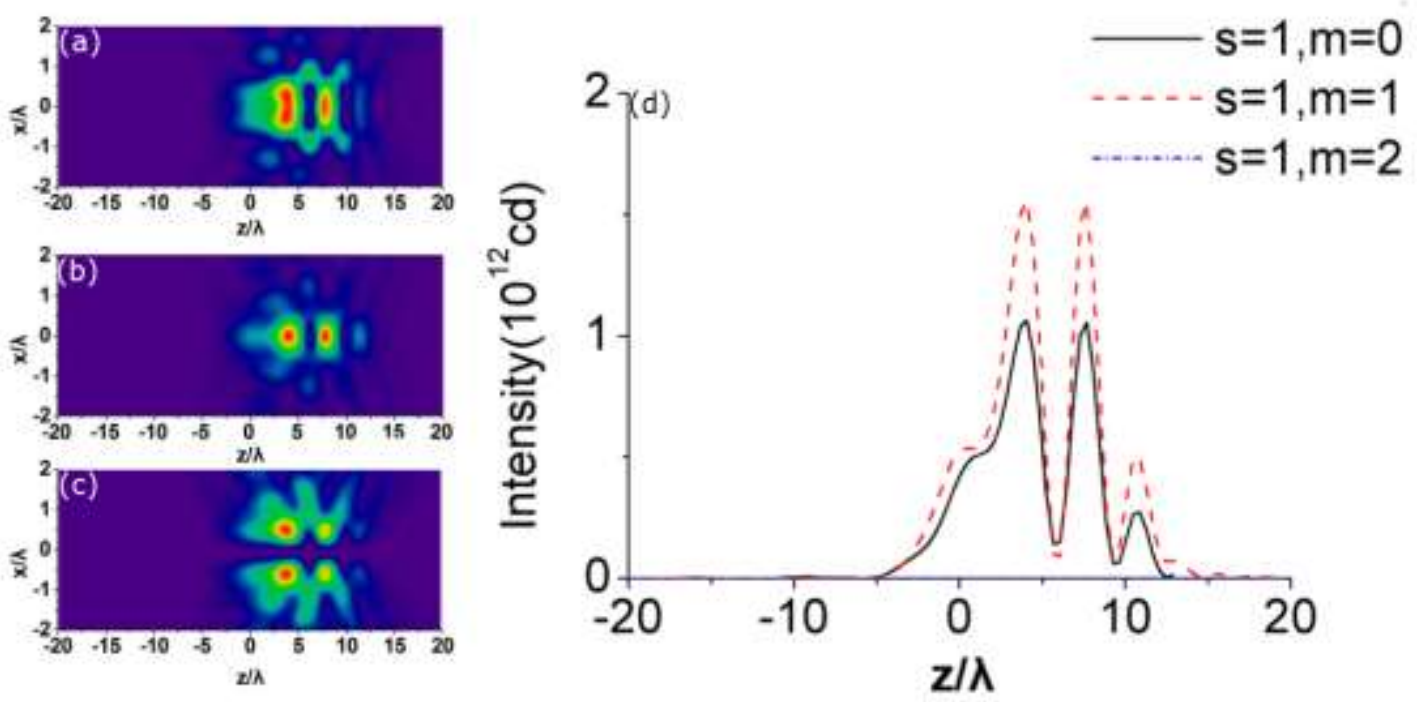

Fig.3 

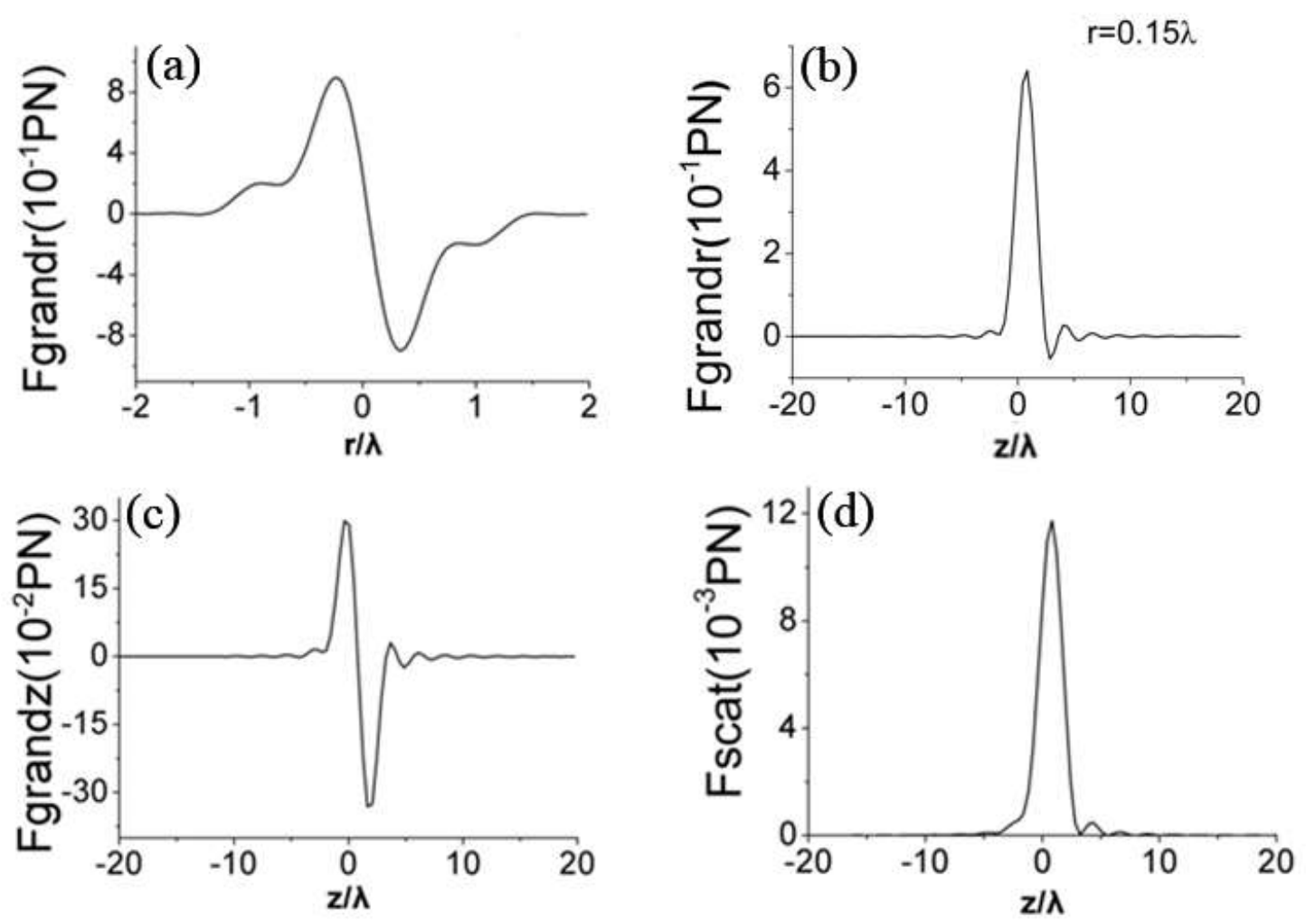

Fig.4 

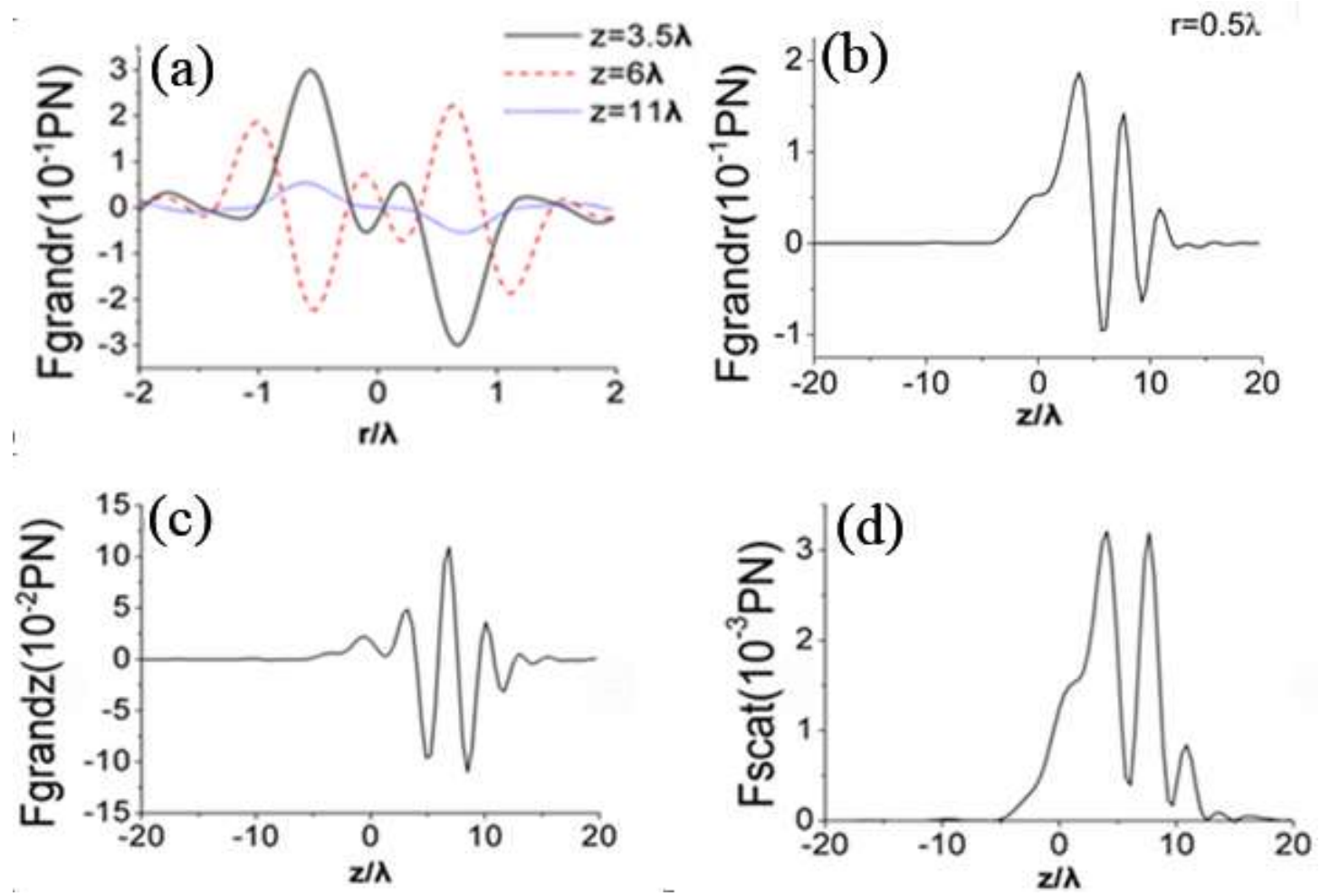

Fig.5 

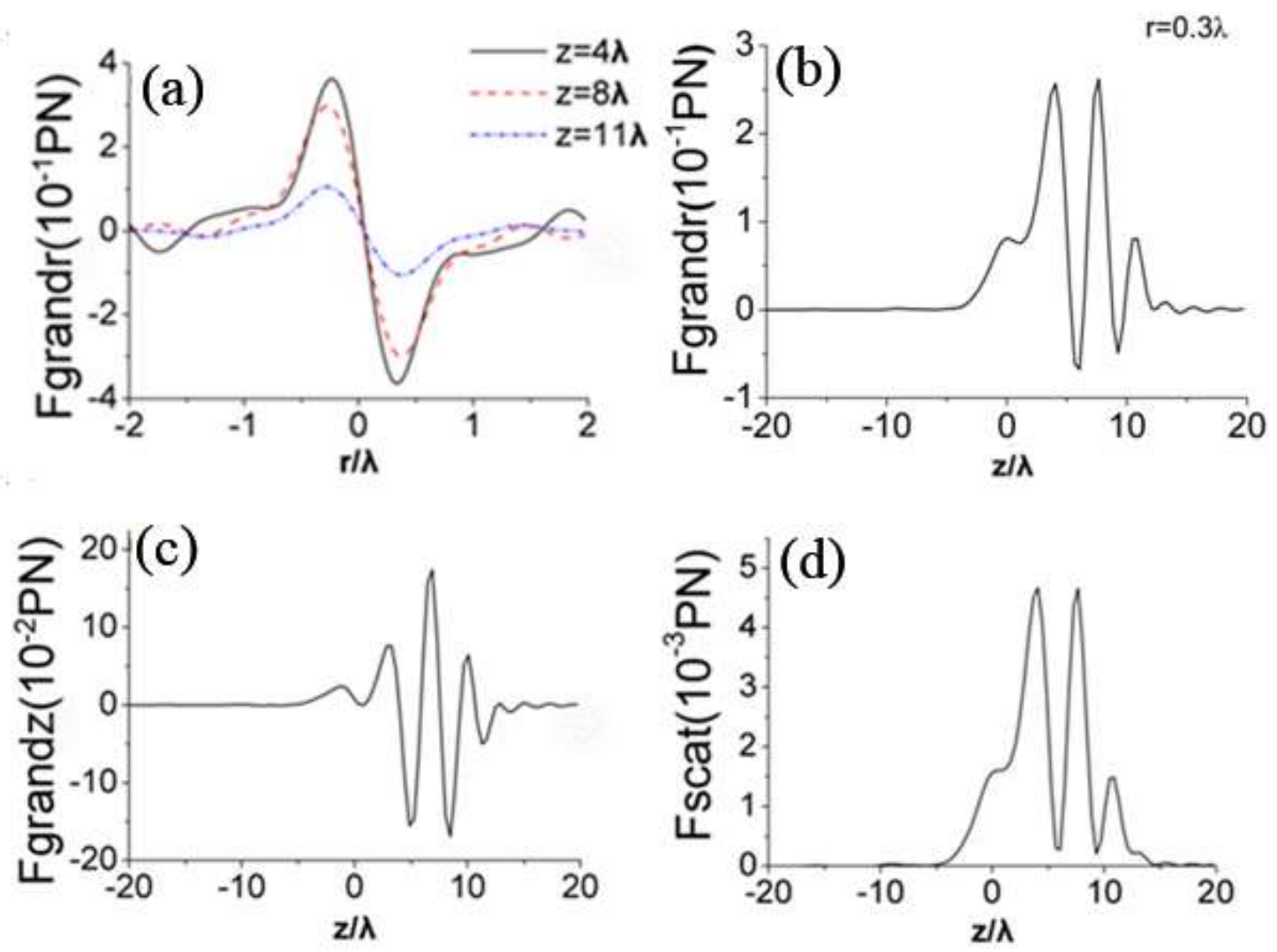

Fig.6 